\title{
LA GESTIÓN DEL CONOCIMIENTO COMO UNA NUEVA CULTURA EMPRESARIAL EN LA ACTUALIDAD
}

\author{
AUTORES: Yanina Holanda Campozano Pilay ${ }^{1}$ \\ Kirenia Maldonado Zúñiga ${ }^{2}$ \\ Leonardo Murillo Quimiz ${ }^{3}$
}

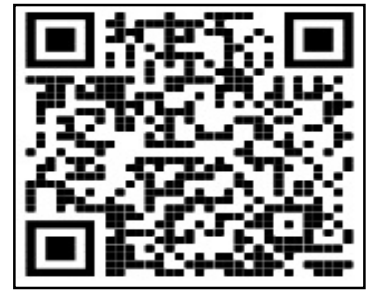

DIRECCIÓN PARA CORRESPONDENCIA: holanda.campozano@unesum.edu.ec

Fecha de recepción: 22/09/2020

Fecha de aceptación: 28/11/2020

\section{RESUMEN}

La gestión del conocimiento existe desde tiempos atrás, pero hoy en día es el auge en la cultura empresarial. Una forma de gestionar las organizaciones que sitúa los recursos humanos como el principal activo y sustenta su poder de competitividad en la capacidad de compartir la información y las experiencias y los conocimientos individuales y colectivos. Es una extraordinaria oportunidad de los profesionales de la información, que pueden aportar sus conocimientos y su experiencia en la explotación adecuada de los recursos externos, la gestión de la información dentro de la organización y la difusión y explotación de la información que ésta genera. Lo que se puede resumir en, información más gestión de recursos humanos. Éste puede ser el binomio del nuevo paradigma de gestión de las corporaciones en los inicios del nuevo siglo. En este contexto, se refiere a una concepción que se comporta en participación, compromiso, motivación y corresponsabilidad de los trabajadores, asociado con las tecnologías de la información y la comunicación, crear las condiciones para el desarrollo de este avance. En la investigación se utilizaron métodos científicos como, histórico - lógico, análisis - síntesis, inducción - deducción y revisión bibliográfica. Los resultados manifestaron las transformaciones positivas que se están adoptando actualmente en las empresas, para que estas puedan lograr un mejor desempeño en las áreas de la organización y mejorar sus ventajas competitivas. Se concluye que este proceso implica actividades sistemática y organizada, al conjunto de la organización, la información corporativa y los conocimientos y opiniones de los trabajadores.

PALABRAS CLAVE: conocimiento; información empresarial; recursos humanos; técnicas de gestión.

\footnotetext{
${ }^{1}$ Universidad Estatal del Sur de Manabí, Facultad de Ciencias Técnicas, Jipijapa, Ecuador. holanda.campozano@unesum.edu.ec Código ORCID: https://orcid.org/0000-0001-5319-6076 2 Universidad Estatal del Sur de Manabí, Facultad de Ciencias Técnicas, kirenia.maldonado@unesum.edu.ec Código ORCID: https://orcid.org/0000-0002-3764-5633

${ }^{3}$ Universidad Estatal del Sur de Manabí, Facultad de Ciencias Técnicas, Jipijapa, Ecuador. leonardo.murillo@unesum.edu.ec.
}

Jipijapa, Ecuador. 
Yanina Holanda Campozano Pilay, Kirenia Maldonado Zúñiga, Leonardo Murillo Quimiz

\title{
KNOWLEDGE MANAGEMENT AS A NEW BUSINESS CULTURE TODAY
}

\begin{abstract}
Knowledge management has existed for a long time, but today it is the boom in business culture, a way of managing organizations that places human resources as the main asset and supports its competitive power in the ability to share information and individual and collective experiences and knowledge. It is an extraordinary opportunity for information professionals, who can contribute their knowledge and experience in the proper exploitation of external resources, the management of information within the organization and the dissemination and exploitation of the information it generates. What can be summarized in, information plus human resources management. This may be the binomial of the new management paradigm for corporations at the beginning of the new century. In this context, refer to a conception that behaves in participation, commitment, motivation and co-responsibility of workers, associated with information and communication technologies, creating the conditions for the development of this advance. The research used scientific methods such as historical - logical, analysis - synthesis, induction deduction and bibliographic review. The results showed the positive transformations that are currently being adopted in companies, so that they can achieve better performance in the areas of the organization and improve their competitive advantages. It is concluded that this process involves systematic and organized activities, the whole of the organization, corporate information and the knowledge and opinions of the workers.
\end{abstract}

KEYWORDS: business information; human resources; management techniques; knowledge

\section{INTRODUCCIÓN}

En las distintas etapas que ha transcurrido la humanidad el conocimiento ha sido la clave para la transformación de la sociedad en general ya que los hechos o información adquiridas por un ente a través de la experiencia o la educación, la comprensión teórica o práctica de un asunto referente a la realidad le permite adquirir o formar un contenido intelectual relativo a un campo determinado o a la totalidad del universo, convirtiéndose todo esto en la base central que le permite al individuo desenvolverse en las diferentes actividades cotidianas y profesionales de su entorno.

Según (Valhondo, 2003) señala que, influido por Sócrates, Plato estaba convencido de que el conocimiento podía alcanzarse. Para él, el conocimiento debía ser certero e infalible y tener como objetivo lo que es en verdad real, en contraste con lo que lo es solo en apariencia. Para ello, distingue entre dos niveles de saber: opinión y conocimiento. Considera opinión las afirmaciones sobre el mundo físico y visible, incluyendo las observaciones y proposiciones de la ciencia; algunas de estas opiniones están bien fundamentadas y otras no, pero ninguna de ellas debe ser entendida como conocimiento verdadero. El conocimiento es el punto más alto del saber por qué concierne a la razón en vez de a la experiencia.

La gestión del conocimiento surge como una nueva cultura empresarial, una manera de gestionar y administrar las organizaciones que sitúa al talento humanos como el principal activo que se sustenta en su poder de creatividad, competitividad en la capacidad de compartir la información, experiencias convirtiéndose en conocimientos individuales y colectivos. 
Muchos investigadores y especialistas han señalado las transformaciones y acontecimientos, que suceden de forma acelerada y contribuyen a la creación de una nueva sociedad, una sociedad que requiere de nuevos saberes, enfoques, tecnologías y nuevos planteamientos para gestionar cualquier tipo de actividad que se desarrolle dentro y fuera de la organización, para crear productos y servicios con un valor agregado que le permita a la organización mantener una ventaja competitiva en el mercado (Pérez Rodríguez, 2005).

De esta forma se abre una extraordinaria oportunidad para la era de la información, donde ahora se pueden aportar conocimientos y experiencias para la adecuada explotación de los recursos externos, la gestión de la información dentro de las organizaciones a través de la difusión y explotación de datos crudos que a su vez ayudarán en la integración de los diversos entes del extracto social.

\section{DESARROLLO}

Tipología de definiciones de Gestión del Conocimiento. Las distintas definiciones de Gestión del Conocimiento que se han propuesto pueden ser integradas en diferentes grupos con se menciona a continuación:

- La Gestión del Conocimiento entendida como la explotación de un recurso a disposición de las diferentes organizaciones y que, si bien, teóricamente, se asume como un recurso intangible, realmente se maneja como un recurso material sin conexión con su creador. El conocimiento existe y está a disposición de las organizaciones, que lo puede mover, almacenar y distribuir sin que exista ningún conflicto o cortapisa (Broadbent, 1997).

- La Gestión del Conocimiento definida desde una perspectiva humana productiva. Donde el humano es el originador del conocimiento, el humano es el poseedor del recurso y el humano es aquel que lo utiliza.

- La Gestión del Conocimiento conceptualizada en base a una descripción del proceso de producción y aplicación del conocimiento.

Definiciones de Gestión del Conocimiento desde una perspectiva de explotación:

- Gestión del Conocimiento es el proceso de identificación, captura y aprovechamiento del conocimiento para ayudar a la compañía a competir. El intercambio y la transferencia son evidencias tangibles de una organización que aprende (O'DELL, 1998).

- La Gestión del Conocimiento es más que la aplicación de TIC’s para gestionar aplicaciones intensivas en conocimiento. Es una nueva manera de pensar sobre las organizaciones modernas. Ayudando a los directivos a poner en relación todos los aspectos de la organización con los temas relacionados con el conocimiento, facilitándoles responder cuestiones tales como cómo apoyar a los trabajadores del conocimiento o cómo transformar el conocimiento en productos y servicios (OWEN, 1999).

- La Gestión del Conocimiento es la suma del capital intelectual, el capital social y los sistemas (SRIKANTAIAH, 2000). 
- La Gestión del Conocimiento se utiliza para permite describir el desarrollo de herramientas, procesos, sistemas, estructuras y culturas para mejorar la creación, compartición y uso del conocimiento crítico para la toma de decisiones (DE LONG, 2000).

- La Gestión del Conocimiento hace referencia a cómo utiliza una organización su capital intelectual (SERENKO, 2002).

Aspectos duales en la Gestión del Conocimiento

Actualmente, tras las distintas concepciones con referencia a cuál es el elemento central de la Gestión del Conocimiento, lo que subyace es, no solo una diferente forma de gestión, sino también diferencias en el objeto de la propia gestión, en este sentido los stocks de conocimiento forman parte de la memoria de la organización, y se relacionan con los repositorios. El objetivo de estos repositorios es capturar conocimiento, ofreciendo un fácil acceso a sus contenidos, con el fin de poner a disposición de los individuos el conocimiento capturado (la información).

Para (DAVENPORT \& DE LONG, 1999) existen tres tipos básicos de repositorios:

- De conocimiento externo (por ejemplo, inteligencia competitiva).

- De conocimiento interno estructurado (informes, material de marketing orientado al producto, metodologías).

- De conocimiento interno informal (por ejemplo: bases de datos de discusiones o "lecciones aprendidas").

Para que pueda haber procesos de innovación sostenibles en el tiempo es necesario que exista un equilibrio entre la disponibilidad y calidad de los datos o información para la actualización o ingreso de nuevos conocimientos, pues sin ellos la información de la que dispone la organización quedaría rápidamente obsoleta (Pérez Rodríguez, 2005).

\section{Los activos de conocimiento}

Los procesos de innovación han concitado mucha atención como elemento fundamental para el éxito y la supervivencia de las empresas de servicios. Al mismo tiempo, existen evidencias empíricas de que los activos de conocimiento están asociados con las actividades de innovación. De este modo, la calidad de la innovación es una función del nivel de activos de conocimiento organizativos. (Ballesteros-Rodríguez, 2014)

El conocimiento adquirido desde el exterior es almacenado como parte de la base de conocimiento de la empresa y es utilizado por aquellos que se implican en el desarrollo de nuevos productos, servicios o procesos. De este modo, la innovación puede verse condicionada por los activos de conocimientos de los que parta la empresa. Sin embargo, muchos de los modelos utilizados para el análisis de los procesos de innovación han tenido un importante sesgo hacia la innovación tecnológica. Además, en muchos casos han partido del supuesto de la existencia de recursos y unidades propias de las empresas de gran tamaño. (Ballesteros-Rodríguez, 2014)

La Gestión del Conocimiento envuelve la identificación y análisis del conocimiento tanto disponible como el requerido, la planeación y control de acciones para desarrollar activos de 
conocimiento, con el fin de alcanzar los objetivos organizacionales, lo que implica básicamente el desarrollo de la gestión estratégica de toda empresa.

Gestión del Conocimiento y Capital Intelectual son dos conceptos que han captado enorme interés, tanto en el mundo empresarial, como académico. Muchas páginas se han escrito sobre la necesidad de relacionar el capital intelectual de la empresa con los objetivos estratégicos, y ahora un número importante de empresas está experimentando con enfoques de gestión del capital intelectual para alcanzar este objetivo. A partir de estos esfuerzos, han surgido diferentes métodos de gestión, medición y elaboración de informes de capital intelectual, con gran relevancia a nivel internacional (Monagas-Docasal, 2012).

Fases y herramientas en la gestión del conocimiento.

Aunque son numerosas las descripciones de los pasos que han de guiar la acción cuando se afronta un proyecto de Gestión del Conocimiento en general se acepta que es necesario:

- Saber cuál es el “conocimiento” que está disponible en la organización.

- Determinar qué “conocimiento” precisa la organización para seguir desarrollando, mejorando o expandiendo sus posibilidades de negocio.

Cuando se haya determinado la disponibilidad y la necesidad de "conocimiento" en un momento dado en la organización, se ha de determinar si el "conocimiento" que se precisa se va a generar dentro de la propia organización o si se va a adquirir en el exterior. Estas decisiones han de estar forzosamente alineadas con la estrategia de la organización.

Una vez adquirido o creado el "conocimiento" este ha de ser convenientemente tratado a fin de que pueda estar disponible para los individuos de la organización que lo precisen.

Paralelamente se ha de dar a conocer la actividad del centro de Gestión del Conocimiento con aquellas acciones de marketing que mejor se adecuen al propio perfil de la organización y de los usuarios potenciales y reales para conseguir el objetivo fundamental que sería la utilización del conocimiento disponible. Este es un ciclo que ha de ser continuamente repetido y, además, concienzudamente evaluado para detectar lagunas o fallos y mejorar su propia dinámica.

La gestión de la información y el conocimiento, las tecnologías, así como las personas que en ello intervienen, existen desde los inicios de la humanidad, cuando el hombre en su afán de comunicación, utilizaba diferentes técnicas para dejar constancia, intercambiar, transmitir y compartir sucesos y apreciaciones de su quehacer y modo de vida, a través de pinturas rupestres, papiros y otros soportes. Esto le permitió de alguna manera gestionar información y por ende generar el conocimiento en sus coterráneos y su descendencia. En la medida en que transcurre el tiempo, aparecen nuevas formas y tecnologías de intercambio producto del desarrollo humano. (Ledo, 2012)

La Gestión de información es el proceso de organizar la información, evaluar, presentar, comparar los datos en un determinado contexto, controlar la calidad, veracidad, que sea oportuna, significativa, exacta y útil y que esté disponible en el momento que se le necesite. Ella se encamina al manejo de la información, documentos, metodologías, informes, publicaciones, soportes y otras actividades en función de los objetivos estratégicos de una organización (Torres Lebrato, 2015). 
En este sentido la gestión de información, es el proceso mediante el cual se obtienen, despliegan o utilizan recursos básicos (económicos, físicos, humanos, materiales) para manejar información dentro y para la sociedad a la que sirve. Tiene como elemento básico, la gestión del ciclo de vida de este recurso y se desarrolla en cualquier organización. En particular, también se desarrolla en unidades especializadas que manejan este recurso en forma intensiva, llamadas unidades de información.

La gestión del conocimiento, son los procesos y acciones de detección, selección, organización, filtrado, presentación y uso de la información por parte de los sectores de una organización. Se encamina al manejo de los recursos humanos (capital humano), formados y preparados para obtener el máximo provecho y emplear la inteligencia colectiva para lograr objetivos estratégicos. De ello se deduce que lo que se gestiona no es el conocimiento, sino las condiciones para que este sea compartido por las personas que lo poseen. (Torres Lebrato, 2015)

De esta forma se concluye que la gestión del conocimiento señala la inclusión de procesos y prácticas de creación, adquisición, captura, reconstrucción y uso de conocimiento, para mejorar el desempeño y la capacidad del aprendizaje en las organizaciones, hecho que se observa en las prácticas de las instituciones analizadas. En la gestión es clave identificar, almacenar y proteger el conocimiento, implícito o explícito, para el futuro operacional y beneficio estratégico de una organización.

\section{Factores de incidencia en la aparición de la Gestión del Conocimiento en las organizaciones}

Resulta una obviedad afirmar que el objetivo primario de cualquier organización con ánimo de lucro es obtener los mejores resultados económicos posibles con el menor coste. Esto se explica porque de esos resultados económicos depende su propia existencia. Por tanto, cuando en un determinado momento se ha producido un avance tecnológico que ha supuesto una mayor eficiencia, ese avance tecnológico se ha convertido en elemento esencial para mantenerse en posiciones económicamente viables. Esto es lo que ha ocurrido con el “conocimiento”. (Rueda Martínez, 2014)

Es importante reconocer la tendencia del valor económico del conocimiento, si bien no es absolutamente novedosa, sí ocupa una posición dominante en los años finales del siglo XX y ello es debido a la disponibilidad de elementos tecnológicos que facilitan su explotación.

Cabe indicar que el disponer de una herramienta tecnológica que posibilite la explotación de un determinado recurso no implica que esa explotación sea llevada a cabo de una manera eficaz y, ni siquiera, que se disponga de la materia prima precisa (se llame esta conocimiento o información).

\section{Sociedad de la Información.}

De acuerdo con (Sánchez-Torres, 2012) el surgimiento de la Sociedad de la Información se da a partir de la convergencia de tres procesos independientes que tienen lugar a finales de los años sesenta y a mediados de los años setenta, los cuales son: la crisis económica y el agotamiento del patrón de acumulación característico del desarrollo industrial, el cual se manifestó con conflictos obreros que llevaron al debilitamiento de la productividad del trabajo, el florecimiento de los movimientos sociales y culturales que cuestionaron la cultura precedente, tales como el feminismo, el ecologismo, el antiautoritarismo, la defensa de los derechos humanos, y la revolución de la tecnología de la información TI; lo cual permitió que se diera una redefinición histórica de las relaciones de poder, de producción y de experiencia sobre las que se basan las

244 UNESUM-Ciencias. Publicación cuatrimestral. Vol. 4, No. 4 (Septiembre-Diciembre), Año 2020. 
sociedades. Cabe destacar, que dichas ideas se enmarcan en fundamentos positivistas, en donde se asocia la mecánica entre el progreso, el bienestar y la ausencia de conflicto como variable funcional.

La sociedad de la información reubica a las tecnologías de la información y de la comunicación, las cuales juegan un rol importante ante las nuevas realidades que viven las instituciones universitarias en lo concerniente a las actividades de docencia, extensión, investigación y gestión; (y) con relación a su posibilidad y capacidad de almacenar, transformar, acceder y difundir información, donde el talento humano es factor fundamental, para el cual se deben promover procesos de aprendizaje permanente que permitan modificar los hábitos de trabajo y conduzcan a enfrentar con éxito los desafíos presentes y futuros. (Pérez Zúñiga, 2018)

En la sociedad de la información, la acción comunicativa y el conjunto de los medios de comunicación de masas (los media) adquieren un renovado papel decisivo en el proceso de construcción del poder. Puesto que los discursos se generan, difunden, debaten, internalizan e incorporan a la acción humana, en el ámbito de la comunicación socializada en torno a las redes locales-globales de comunicación, las redes de comunicación y nuestra actuación en y a partir de ellas, resultará clave en la definición de las relaciones de poder en nuestros días. (Pérez Zúñiga, 2018)

Los activos intangibles la puesta en valor de los llamados "activos intangibles" surge de una evidencia económica: el exceso de valor de mercado de ciertas compañías en relación con el valor reflejado en los estados financieros.

El valor de una marca, la confianza de los consumidores en ella, la reputación de una compañía, las relaciones establecidas con distribuidores y/o proveedores son elementos que suponen un diferencial con respecto a otras organizaciones competidoras. La apreciación de los activos intangibles deriva, pues, del reconocimiento de que, en las economías más desarrolladas, la producción de bienes y/o servicios involucraba no solo los activos tradicionales (materias primas, mano de obra o capital), sino también elementos como: los valores y cultura organizacionales; el valor de la marca, su reputación y credibilidad; las metodologías y sistemas de trabajo; el entramado de relaciones (con clientes, proveedores, administraciones) o las habilidades, destrezas y conocimiento de los empleados (Pérez Rodríguez, 2005).

De una forma muy general, podíamos definir los activos intangibles como todo aquello que, en principio, queda fuera de las contabilidades empresariales, pero conforma el valor real de mercado de una compañía. Las reingenierías de procesos de negocio.

En las dos últimas décadas se ha observado un creciente interés por los activos intangibles, componente invisible de las empresas y la economía en general. Es tarea de las autoridades competentes, las universidades e investigadores la máxima divulgación de una disciplina cuya materia prima es la medición y/o valoración del conocimiento contenido en las organizaciones y en las personas que las conforman (CITRARO, 2010).

Sociedad de la Información o Sociedad del Conocimiento

La sociedad del conocimiento es la sociedad que ha empezado a emerger en el mundo y que se tiene como meta construir para resolver los grandes problemas de la humanidad, como la violencia en todas sus manifestaciones, la destrucción del ambiente ecológico, la corrupción, el 
desempleo, la desnutrición, la baja calidad de vida que afecta a muchas poblaciones, etcétera, mediante la búsqueda, procesamiento, adaptación, creación, innovación y aplicación del conocimiento a través de diferentes medios (Tobón, 2013).

La información, la comunicación, la educación y el conocimiento son esenciales para la iniciativa, el progreso y el bienestar de las sociedades. A su vez, las tecnologías de la información y la comunicación (TIC) que potencian esos cuatro conceptos, tienen inmensas repercusiones en prácticamente todas las dimensiones de nuestras vidas. La capacidad de las tecnologías digitales para superar o, en todo caso, reducir las consecuencias de muchos obstáculos tradicionales, especialmente los que suponen el tiempo y la distancia, ha propiciado que, por primera vez en la historia, el vasto potencial de estas tecnologías sea utilizado por millones de personas en todo el mundo y en beneficio de ellas (García Aretio, 2012).

\section{Tendencias en Gestión del Conocimiento}

Para esta investigación se consideró el análisis de las distintas perspectivas desde las que se aborda la gestión del conocimiento, ya que estas perspectivas, consolidadas como tendencias, suponen planteamientos diferenciados con respecto al alcance y naturaleza de la gestión del conocimiento.

La visión económica este apartado capta el interés de las empresas lo que hace relevante la Gestión del Conocimiento como alternativa en la búsqueda de ventajas competitivas estables y duraderas.

El interés en los aspectos ligados al valor económico y a su forma de medición es el elemento central de la visión económica de la GC, e implica una preocupación por un adecuado conocimiento de los activos intangibles para conseguir gestionarlos eficientemente y alcanzar los objetivos presentes de la organización y determinar los objetivos futuros. Los activos intangibles deben ser, al igual que los tangibles, utilizados y explotados convenientemente. (Pérez Rodríguez, 2005)

(Rincón, 2017) opina que gracias a este aporte y a las nuevas tendencias de la época orientadas a la apertura económica y la competitividad, muchas organizaciones vieron la necesidad de incorporar esta visión al desarrollo de las áreas de gestión de recursos humanos, dando lugar a la identificación de las competencias requeridas por los individuos en los procesos de selección, así como su desarrollo durante su permanencia en la organización, mediante el diseño e implementación de programas de capacitación permanentes para la promoción del talento humano.

\section{Herramientas de medición del conocimiento}

Según (Pérez Rodríguez, 2005) Estas herramientas son intentos para poner en valor contable aspectos clave de la dinámica empresarial actual, para proporcionar medidas del rendimiento organizacional que, con criterios más amplios, incluyan más perspectivas que las financieras. No obstante, los planteamientos difieren de unas a otras y, de una manera muy genérica, podemos decir que buscan:

Determinar y describir los elementos que forman parte de los activos intangibles.

Medir los distintos elementos. Describir como los elementos se relacionan y proporcionan valor. 
En la actualidad, las organizaciones de información han comenzado a insertarse de manera intensiva en los temas de medición, aunque solamente identifican determinados indicadores en segmentos críticos de la organización para medir aspectos o áreas puntuales. No existe, por tanto, un modelo para la medición del conocimiento, aunque es posible aplicar los modelos precursores en estas organizaciones tomando en cuenta sus propias particularidades. De ahí la oportunidad de contar con un modelo que se base en un pensamiento integral para la medición de la gestión del conocimiento como macroproceso estratégico, en función de lograr resultados de calidad en el comportamiento y rendimiento de las organizaciones de información que gestionan conocimiento. (León Santos, 2011)

\section{La visión tecnológica}

(Prado, 2013) señala que se evidencia que la generación de las capacidades dinámicas, a partir de los procesos de exploración, explotación y ambidestreza organizacional, requiere de un contexto adecuado. Para ello, la empresa debe conocerse a sí misma, para poder extraer el mayor valor posible de los conocimientos que residen en los miembros de la organización y en los diferentes niveles organizacionales. Razón por la que se hace necesario crear a través de la gestión eficiente del conocimiento un contexto en el que los intangibles disponibles en una organización sean accesibles y se puedan utilizar para el desarrollo de las capacidades y competencias requeridas por la organización para facilitar una rápida adaptación de la empresa a los cambios del entorno y, que a su vez, permitan la generación de innovación tecnológica y mejorar la toma de decisiones.

Metodología de la gestión del conocimiento es el Procedimiento para la implantación de la gestión del conocimiento en una empresa:

- Definir el negocio en términos de conocimiento.

- Esclarecer cómo se compone el capital intelectual de la empresa.

- Identificar aquellos empleados que producen los conocimientos que dan ventaja competitiva a la empresa.

- Convertir el conocimiento generado por los empleados de desempeño superior en información: clasificarla, distribuirla y hacerla accesible.

- Identificar las competencias que permiten a los empleados de desempeño superior utilizar la información de manera inteligente.

- Impulsar un cambio cultural para que los talentos compartan sus conocimientos.

\section{Con la gestión del conocimiento una organización puede tener varios beneficios como:}

Uso cada vez más intensivo del conocimiento en la producción de bienes y servicios, a diferencia de la era industrial cuando lo predominante era la utilización de capital o mano de obra.

Desarrollo de tecnología e información para ser más competitivos, este crecimiento sólo puede provenir de la innovación en productos y servicios. 
Yanina Holanda Campozano Pilay, Kirenia Maldonado Zúñiga, Leonardo Murillo Quimiz

Aumento en el flujo de conocimiento, al utilizar las redes informáticas se eliminan demoras y se utiliza y comparte la información rápidamente entre fabricantes, proveedores, trabajadores y clientes.

Desarrollo de smartproducts: los productos actuales concentran cada vez más conocimiento y, por lo tanto, deben ser cada vez más inteligentes.

\section{MATERIALES Y MÉTODOS}

La metodología de la investigación científica permitió el desarrollo de la investigación, a través de los métodos científicos se definió y sistematizó los procedimientos a seguir durante el proceso del estudio para analizar la gestión del conocimiento y sus transformaciones en la sociedad.

En la investigación se utilizaron métodos de investigación científica tales como:

Del nivel teórico los métodos:

Histórico - lógico: se utilizó en la construcción de la investigación con énfasis en la búsqueda de los antecedentes relacionados a la gestión del conocimiento.

Análisis - síntesis: se usó para analizar y determinar los criterios de las fases y herramientas en la gestión del conocimiento.

Inducción - deducción: se empleó en toda la investigación, en particular en las características y avances de la gestión del conocimiento en la actualidad.

Del nivel Empírico:

Revisión bibliográfica: Se utilizó para la recopilación de información relacionada al tema, mediante libros, revistas de carácter científico, internet, entre otros.

\section{RESULTADOS}

La gestión del conocimiento ha transformado la forma en que muchas empresas trabajan en la actualidad, estas pueden crear y difundir información vital de una manera sistemática y eficiente con el fin de lograr un mejor desempeño en las áreas de la organización y mejorar sus ventajas competitivas. Las organizaciones se fundamentan en dos procesos, el primero, es el desarrollo de ambientes de aprendizaje que facilitan la creación de conocimiento y el segundo, es lograr el impulso de la interacción del conocimiento. Este proceso permite, a su vez, Identificar los conocimientos que necesita la organización. Luego de adquirir los conocimientos, los aplica de forma efectiva a través de los procesos estratégicos: identificación, adquisición, desarrollo, distribución, uso, retención y medición.

Es importante conocer los dos tipos de conocimiento, el explícito y el conocimiento tácito, estos permiten planificar y estructurar los cambios, eligiendo correctamente las técnicas y las herramientas adecuadas para cada empresa. El conocimiento explícito es el que se encuentra estructurado, tiene forma y es sistemático. Es el conocimiento articulado expresado y registrado con palabras, números, imágenes, códigos, principios universales, fórmulas científicas, etc. Su información está habitualmente almacenada en documentos, procedimientos, programas, procesos, manuales, especificaciones, tutoriales o en bases de datos. Es fácil de compartir y comunicar de un individuo a otro, muchas veces se encuentra esquematizado para facilitar su difusión. 
El conocimiento tácito se construye poco a poco, sin tener conciencia de ello, este es el resultado de la experiencia, de los aprendizajes, de los hábitos que van acumulando a lo largo de la vida, e involucra elementos tales como la intuición, creencias, costumbres, valores, o puntos de vista. Se encuentra en un nivel del inconsciente y aparece cuando lo necesitamos. Este conocimiento es altamente personal o característico de un grupo, por eso en ocasiones resulta muy difícil de explicar o comunicar a los demás. Este tipo de conocimiento no se puede estructurar, almacenar ni distribuir y es muy difícil de gestionar.

Se debe identificar qué elementos del conocimiento tácito pueden ser capturados para poder hacerlos explícitos y codificarlos, de esta forma se puede compartir con las otras personas. Las organizaciones se encuentran en continuo movimiento y crecimiento, es así como aparece el conocimiento como herramienta estratégica, esto es un arma privilegiada para mejorar el rendimiento y optimizar la información.

Para lograr esto se necesitan nuevas tecnologías y herramientas, que conduzcan a gestionar eficazmente el conocimiento de las organizaciones. No es suficiente con que se tengan claras las ideas sobre tendencias y estrategias productivas. La interrogante es: ¿Cómo gestionar el conocimiento de la empresa?, ¿Cómo agilizar los procesos de gestión de información? Los de datos almacenados en un cerebro esconden un estratégico valor oculto del que no se puede ni se sabe prescindir. Existen avanzadas herramientas, que permiten descubrir el perfil de los clientes y conocer las relaciones ocultas entre ellas y sus preferencias.

Algunas técnicas como Data Mart, o los sistemas de Gestión Documental son algunas de las herramientas tecnológicas para facilitar la gestión del conocimiento, y así tener un sistema que agilice y mejore la toma de decisiones estratégicas. La gestión del conocimiento, debe de ser un proceso constante si una empresa desea crecer y mantenerse en el mercado. Debe intuir, el conocimiento, pues este es parte esencial del ser humano, las investigaciones sobre el papel que juega dentro de las organizaciones y las economías tiene un amplio repertorio de consecuencias en la teoría de la dirección y más ampliamente en la teoría económica.

Se conoce que las personas son el centro de la gestión del conocimiento. Pero lo que es importante para esta herramienta son sus habilidades y su información. Estos dos componentes son básicos a la hora de tener en cuenta el proceso de gestión del conocimiento en una organización. La información puede ser recogida tratada y almacenada por los sistemas de información de la empresa facilitando la creación de un cuadro de mando del entorno y un cuadro de mando integral para la dirección. Si estos sistemas están bien diseñados se obtendrá la información periódica y sistemática de lo que ocurre tanto dentro como fuera de la organización y permite tomar decisiones con rapidez.

El pensamiento humano puede categorizarse en tres niveles: datos, información y conocimiento, cierto volumen de datos organizados o procesados pueden constituir información, pero cuando la información es almacenada en papel, archivos, bases de datos (conocimiento explícito) que nadie lee o usa y cuando esa cantidad de información crece, se vuelve complicado encontrar lo que es realmente valioso. La información puede convertirse en algo valioso cuando es usada dentro de un cierto contexto para tomar una acción de manera oportuna y acertada. Es entonces cuando se puede hablar de conocimiento. 
Las organizaciones actuales normalmente almacenan datos mediante el uso de tecnologías. Desde un punto de vista cuantitativo, las empresas evalúan la gestión de los datos en términos de costo, velocidad y capacidad. Todas las organizaciones necesitan datos y algunos sectores son totalmente dependientes de ellos. Bancos, compañías de seguros, agencias gubernamentales y la seguridad social son ejemplos obvios. En este tipo de organizaciones la buena gestión de los datos es esencial para su funcionamiento, pues operan con millones de transacciones diarias.

Pero en general, para la mayoría de las empresas tener muchos datos no siempre es bueno. Las organizaciones almacenan datos sin sentido. Realmente esta actitud no tiene sentido por dos razones. La primera es que demasiados datos hacen más complicado identificar aquellos que son relevantes. La toma de decisiones de una organización se basa en datos, pero estos nunca dirán lo que hay que hacer pero estos son importantes para las organizaciones, pues son la base para la creación de información y nuevas estrategias.

Las habilidades son un cambio más complicadas de trasmitir, estas implican adquirir nuevos conocimientos. Estas se asocian con los planes de formación y supone un esfuerzo muy importante identificar aquellas personas que poseen las habilidades más valiosas dentro y fuera de la organización para que compartan sus conocimientos. Las habilidades e informaciones útiles se transmiten entre los empleados de forma rápida y de esta forma aumenta la posibilidad de generar nuevo conocimiento que deriva en aplicaciones nuevas, mejoras en procesos o productos y nuevas formas de hacer negocio para alcanzar nuevas oportunidades.

El conocimiento es el único activo que crece con el tiempo y no se desgasta pero que puede desaparecer con las personas, si este no es compartido. Existen casos en los que un empleado se va de una organización al no encontrar crecimiento personal, por eso es vital gestionarlo, tanto en grandes organizaciones como en pequeñas organizaciones.

Por último, la gestión del conocimiento es también considerada como una herramienta que permite la integración de la globalidad de la organización, replanteando de manera continua y dinámica la redefinición de la misión de la organización, es esencial para favorecer una estructura empresarial innovadora y eficiente.

Si el conocimiento fluye y se transmite de manera correcta en la organización, este solo puede crecer.

\section{CONCLUSIONES}

La gestión del conocimiento, es un proceso que implica distintas problemáticas, una se encuentra en que su implantación sea eficaz. En segundo lugar, los costos son un motivo de dificultad a la hora de implantar la gestión del Conocimiento. En tercer lugar, los problemas de integrar y motivar a la plantilla de colaboradores. Le siguen la resistencia al cambio y por último la dificultad en el desarrollo y costo de la tecnología. Estos obstáculos pueden disuadir a las organizaciones de su uso, se debe de pensar que a través de su implantación se invierte directamente e indirectamente en el principal recurso de las compañías, el capital intelectual.

El desarrollo del capital humano es el resultado de los beneficios que brindan los procesos de capacitación y desarrollo en las organizaciones. Es necesario instrumentar a nivel organizacional políticas adecuadas de capacitación y desarrollo de recursos humanos con el objetivo de mantener, incrementar, perfeccionar y aprovechar a niveles más altos los conocimientos esenciales que requiere la organización para su funcionamiento y desempeño.

250 UNESUM-Ciencias. Publicación cuatrimestral. Vol. 4, No. 4 (Septiembre-Diciembre), Año 2020. 
La razón por la que existe una organización es para cumplir con su propósito fundamental, una empresa existe para satisfacer una necesidad del mercado. La organización nunca es un fin, sino un medio para llegar a él. La organización debe seguir y nunca preceder al negocio, por lo tanto no es posible que exista una organización basada en el conocimiento si no existe antes un negocio basado en el conocimiento.

Es importante definir un negocio basado en el conocimiento consiste en identificar el negocio en términos de lo que la empresa "conoce”, es decir, la forma en que utiliza la información para actuar. La empresa puede saber acerca de sus clientes, de un producto o de cierta tecnología. El desafío que supone identificar ese saber consiste en ir más allá de lo tangible y definir el negocio en términos de su esencia.

\section{REFERENCIAS BIBLIOGRÁFICAS}

Ballesteros-Rodríguez, J. L.-D.-A. (2014). Los procesos de innovación y los activos de conocimiento en las empresas de restauración. MANAGEMENT STUDIES INTERNATIONAL CONFERENCE.

Ballesteros-Rodríguez, J. L.-D.-A. (2014). Los procesos de innovación y los activos de conocimiento en las empresas de restauración. MANAGEMENT STUDIES INTERNATIONAL CONFERENCE.

Broadbent, M. (1997). The emerging phenomenon of knowledge management. Australian Library Journal, 6-24.

Citraro, L. T. (2010). Los activos intangibles dentro del contexto de la sociedad del conocimiento: el reto de su identificación y valoración. Propiedad Intelectual, 9(13).

Davenport, T. H., \& DE LONG, D. W. (1999). Successful knowledge management projects. Boston: ButterworthHeinemann, 89-107.

De Long, D. y. (2000). Confronting conceptual confusion and conflict in knowledge management. Organizational Dynamics, 29(1).

García Aretio, L. (2012). Nuevo libro en línea:“Sociedad del conocimiento y educación”. Contextos Universitarios Mediados, 4.

Ledo, M. J. (2012). Gestión de la información y el conocimiento. Revista Cubana de educación médica superior, 26(3).

León Santos, M. \&. (2011). Propuesta de un modelo de medición para los procesos de la gestión del conocimiento en organizaciones de información. Revista Interamericana de Bibliotecología, 34(1).

Monagas-Docasal, M. (2012). El capital intelectual y la gestión del conocimiento. Ingeniería Industrial, 33(2).

O'Dell, C. y. (1998). If only we knew what we know: Identification and transfer of internal best practices. California Management Review, 40(3).

Owen, J. M. (1999). Knowledge management and the information. Information Services \& Use, 19(1).

Pérez Rodríguez, Y. \&. (2005). La gestión del conocimiento: un nuevo enfoque en la gestión empresarial. Acimed, 13(6).

Pérez Zúñiga, R. M. (2018). La sociedad del conocimiento y la sociedad de la información como la piedra angular en la innovación tecnológica educativa. RIDE. Revista Iberoamericana para la Investigación y el Desarrollo Educativo, 8(16).

Prado, J. C.-S. (2013). Capacidades dinámicas y gestión del conocimiento en nuevas empresas de base tecnológica. Cuadernos de administración, 26(47).

Rincón, R. A. (2017). Gestión del conocimiento y aprendizaje organizacional: una visión integral. Informes Psicológicos, 17(1). 
Rueda Martínez, M. I. (2014). La Gestión del Conocimiento y la Ciencia de la Información: Relaciones disciplinares y profesionales.

Sánchez-Torres, J. M.-Z. (2012). La sociedad de la información: génesis, iniciativas, concepto y su relación con las TIC. Revista UIS Ingenierías, 11(1).

Serenko, A. y. (2002). Global ranking of knowledge management and intellectual capital academic journals. Journal of Knowledge Management, 13(1).

Srikantaiah, K. (2000). Knowledge management: A faceted overview. Information Today, 1-17.

Tobón, S. (2013). Los proyectos formativos: transversalidad y desarrollo de competencias para la sociedad del conocimiento. México D.F.: Instituto CIFE, 33.

Torres Lebrato, L. (2015). La gestión de información y la gestión del conocimiento. Revista Archivo Médico de Camagüey, 19(2).

Valhondo, D. (2003). Gestión del conocimiento: del mito a la realidad. Ediciones Díaz de Santos. 